International Journal of Pure and Applied Mathematics

Volume 107 No. 3 2016, 773-788

ISSN: 1311-8080 (printed version); ISSN: 1314-3395 (on-line version)

url: http://www.ijpam.eu

doi: 10.12732/ijpam.v107i3.23

\title{
SOFT $L$-FUZZY PRE-UNIFORM SPACES INDUCED BY SOFT $L$-FUZZY TOPOGENOUS ORDERS
}

\author{
Yong Chan Kim \\ Department of Mathematics \\ Gangneung-Wonju University \\ Gangneung, Gangwondo, 210-702, KOREA
}

\begin{abstract}
In this paper, we introduce the notions of soft $L$-fuzzy pre-uniformities and soft $L$-fuzzy topogenous orders in complete residuated lattices. We study some relationships between previous spaces. As main results, we investigate the soft $L$-fuzzy pre-uniformities (resp. soft $L$-quasi-uniformities) induced by soft $L$-fuzzy topogenous orders (resp. $\operatorname{soft} L$ fuzzy topogenous structure). We give their examples.
\end{abstract}

AMS Subject Classification: 03E72, 06A15, 06F07, 54A05

Key Words: complete residuated lattice, soft $L$-quasi-uniformities, soft $L$-fuzzy topogenous orders

\section{Introduction}

Hájek [4] introduced a complete residuated lattice which is an algebraic structure for many valued logic. It is an important mathematical tool for algebraic structures [5,7-9]. Recently, Molodtsov [11] introduced the soft set as a mathematical tool for dealing information as the uncertainty of data in engineering, physics, computer sciences and many other diverse field. Presently, the soft set theory is making progress rapidly $[1,3]$. Pawlak's rough set $[12,13]$ can be viewed as a special case of soft rough sets [3]. The topological structures of soft sets have been developed by many researchers [2,7-9,14-17].

Ko [7] introduced a fuzzy soft $F: A \rightarrow L^{U}$ as an extension as the soft $F$ :

Received: January 23, 2016

Published: April 22, 2016
(C) 2016 Academic Publications, Ltd.

url: www.acadpubl.eu 
$A \rightarrow P(U)$ where $L$ is a complete residuated lattice. Ko [7-9] introduced the soft topological structures, $L$-fuzzy quasi-uniformities and soft $L$-fuzzy topogenous orders in complete residuated lattices.

In this paper, we introduce the notions of soft $L$-fuzzy pre-uniformities and soft $L$-fuzzy topogenous orders in complete residuated lattices. We study some relationships between previous spaces. As main results, we investigate the soft $L$-fuzzy pre-uniformities (resp. soft $L$-fuzzy quasi-uniformities) induced by soft $L$-fuzzy topogenous orders (resp. soft $L$-fuzzy topogenous structure). We give their examples.

\section{Preliminaries}

Definition 1. [4,5] An algebra $(L, \wedge, \vee, \odot, \rightarrow, 0,1)$ is called a complete residuated lattice if it satisfies the following conditions:

(C1) $L=(L, \leq, \vee, \wedge, 1,0)$ is a complete lattice with the greatest element 1 and the least element 0 ;

(C2) $(L, \odot, 1)$ is a commutative monoid;

(C3) $x \odot y \leq z$ iff $x \leq y \rightarrow z$ for $x, y, z \in L$.

In this paper, we assume that $\left(L, \leq, \odot, \rightarrow, \oplus,^{*}\right)$ is a complete residuated lattice with an order reversing involution * which is defined by $x \oplus y=$ $\left(x^{*} \odot y^{*}\right)^{*}$ and $x^{*}=x \rightarrow 0$.

Lemma 2. $[4,5]$ For each $x, y, z, x_{i}, y_{i}, w \in L$, we have the following properties.

(1) $1 \rightarrow x=x, 0 \odot x=0$,

(2) If $y \leq z$, then $x \odot y \leq x \odot z, x \oplus y \leq x \oplus z, x \rightarrow y \leq x \rightarrow z$ and $z \rightarrow x \leq y \rightarrow x$

(3) $x \odot y \leq x \wedge y \leq x \vee y \leq x \oplus y$,

(4) $\left(\bigwedge_{i} y_{i}\right)^{*}=\bigvee_{i} y_{i}^{*},\left(\bigvee_{i} y_{i}\right)^{*}=\bigwedge_{i} y_{i}^{*}$,

(5) $x \odot\left(\bigvee_{i} y_{i}\right)=\bigvee_{i}\left(x \odot y_{i}\right)$,

(6) $x \oplus\left(\bigwedge_{i} y_{i}\right)=\bigwedge_{i}\left(x \oplus y_{i}\right)$,

(7) $x \rightarrow\left(\bigwedge_{i} y_{i}\right)=\bigwedge_{i}\left(x \rightarrow y_{i}\right)$,

(8) $\left(\bigvee_{i} x_{i}\right) \rightarrow y=\bigwedge_{i}\left(x_{i} \rightarrow y\right)$, 
(9) $x \rightarrow\left(\bigvee_{i} y_{i}\right) \geq \bigvee_{i}\left(x \rightarrow y_{i}\right)$,

(10) $\left(\bigwedge_{i} x_{i}\right) \rightarrow y \geq \bigvee_{i}\left(x_{i} \rightarrow y\right)$,

(11) $(x \odot y) \rightarrow z=x \rightarrow(y \rightarrow z)=y \rightarrow(x \rightarrow z)$,

(12) $x \odot(x \rightarrow y) \leq y$ and $x \rightarrow y \leq(y \rightarrow z) \rightarrow(x \rightarrow z)$,

(13) $(x \rightarrow y) \odot(z \rightarrow w) \leq(x \odot z) \rightarrow(y \odot w)$,

(14) $(x \rightarrow y) \odot(z \rightarrow w) \leq(x \oplus z) \rightarrow(y \oplus w)$,

$(15) x \rightarrow y \leq(x \odot z) \rightarrow(y \odot z)$ and $(x \rightarrow y) \odot(y \rightarrow z) \leq x \rightarrow z$,

(16) $x \odot y \odot(z \odot w) \leq(x \odot z) \oplus(y \odot w)$.

(17) $x \rightarrow y=y^{*} \rightarrow x^{*}$.

Definition 3. [7-9] Let $X$ be an initial universe of objects and $E$ the set of parameters (attributes) in $X$. A pair $(F, A)$ is called a fuzzy soft set over $X$, where $A \subset E$ and $F: A \rightarrow L^{X}$ is a mapping. We denote $S(X, A)$ as the family of all fuzzy soft sets under the parameter $A$.

Definition 4. [7-9] Let $(F, A)$ and $(G, A)$ be two fuzzy soft sets over a common universe $X$.

(1) $(F, A)$ is a fuzzy soft subset of $(G, A)$, denoted by $(F, A) \leq(G, A)$ if $F(\epsilon) \leq G(\epsilon)$, for each $\epsilon \in A$.

(2) $(F, A) \wedge(G, A)=(F \wedge G, A)$ if $(F \wedge G)(\epsilon)=F(\epsilon) \wedge G(\epsilon)$ for each $\epsilon \in A$.

(3) $(F, A) \vee(G, A)=(F \vee G, A)$ if $(F \vee G)(\epsilon)=F(\epsilon) \vee G(\epsilon)$ for each $\epsilon \in A$.

(4) $(F, A) \odot(G, A)=(F \odot G, A)$ if $(F \odot G)(\epsilon)=F(\epsilon) \odot G(\epsilon)$ for each $\epsilon \in A$.

(5) $(F, A)^{*}=\left(F^{*}, A\right)$ if $F^{*}(\epsilon)=(F(\epsilon))^{*}$ for each $\epsilon \in A$.

(6) $(F, A) \oplus(G, A)=(F \oplus G, A)$ if $(F \oplus G)(\epsilon)=\left(F^{*}(\epsilon) \odot G^{*}(\epsilon)\right)^{*}$ for each $\epsilon \in A$.

Definition 5. [9] A mapping $\xi: S(X, A) \times S(X, A) \rightarrow L$ is called a soft $L$-fuzzy semi-topogenous order on $(X, A)$ if it satisfies the following axioms.

(ST1) $\xi\left(\left(1_{X}, A\right),\left(1_{X}, A\right)\right)=\xi\left(\left(0_{X}, A\right),\left(0_{X}, A\right)\right)=1$.

(ST2) If $\xi((F, A),(G, A)) \neq 0$, then $(F, A) \leq(G, A)$. 
(ST3) If $\left(F_{1}, A\right) \leq(F, A), \quad(G, A) \leq\left(G_{1}, A\right)$, then $\xi((F, A),(G, A)) \leq$ $\xi\left(\left(F_{1}, A\right),\left(G_{1}, A\right)\right)$.

A mapping $\xi$ is called a soft strong $L$-fuzzy semi-topogenous order on $(X, A)$ if it satisfies (ST1), (ST3) and the following axiom.

(S) $\xi((F, A),(G, A)) \leq S((F, A),(G, A))$ where

$$
S((F, A),(G, A))=\bigwedge_{a \in A} \bigwedge_{x \in X}(F(a)(x) \rightarrow G(a)(x)) .
$$

Remark 6. If $\xi$ is a soft (resp. strong) $L$-fuzzy semi-topogenous order on $(X, A)$. Define a mapping $\xi^{s}: S(X, A) \times S(X, A) \rightarrow L$ as $\xi^{s}((F, A),(G, A))=$ $\xi\left((G, A)^{*},(F, A)^{*}\right)$. Then $\xi^{s}$ is a soft (resp. strong) $L$-fuzzy semi-topogenous order on $(X, A)$.

Definition 7. [9] A soft (resp. strong) $L$-fuzzy semi-topogenous order $\xi$ is called:

(1) soft (resp. strong) $L$-fuzzy topogenous if ( $\mathrm{T}$ )

$$
\begin{aligned}
& \xi\left(\left(F_{1}, A\right) \odot\left(F_{2}, A\right),\left(G_{1}, A\right) \odot\left(G_{2}, A\right)\right) \\
& \geq \xi\left(\left(F_{1}, A\right),\left(G_{1}, A\right)\right) \odot \xi\left(\left(F_{2}, A\right),\left(G_{2}, A\right)\right) .
\end{aligned}
$$

(2) soft (resp. strong) $L$-fuzzy cotopogenous if (CT)

$$
\begin{aligned}
& \xi\left(\left(F_{1}, A\right) \oplus\left(F_{2}, A\right),\left(G_{1}, A\right) \oplus\left(G_{2}, A\right)\right) \\
& \geq \xi\left(\left(F_{1}, A\right),\left(G_{1}, A\right)\right) \odot \xi\left(\left(F_{2}, A\right),\left(G_{2}, A\right)\right),
\end{aligned}
$$

(3) soft (resp. strong) $L$-fuzzy bitopogenous if $\xi$ are soft (resp. strong) $L$-fuzzy topogenous and soft (resp. strong) $L$-fuzzy cotopogenous.

Definition 8. [9] A soft (resp. strong) L-fuzzy topogenous (resp. cotopogenous) order $\xi$ on $(X, A)$ is said to be a soft (resp. strong) $L$-fuzzy topogenous (resp. cotopogenous) structure if $\xi \circ \xi \geq \xi$, where

$$
\begin{aligned}
& (\xi \circ \xi)((F, A),(H, A)) \\
& =\bigvee_{(G, A) \in S(X, A)} \xi((F, A),(G, A)) \odot \xi((G, A),(H, A))
\end{aligned}
$$

Definition 9. [9] A mapping $\mathcal{U}: S(X \times X, A) \rightarrow L$ is called a soft $L$-fuzzy pre-uniformity on $X$ iff it satisfies the properties.

(SU1) There exists $(U, A) \in S(X \times X, A)$ such that $\mathcal{U}((U, A))=1$,

(SU2) If $(V, A) \leq(U, A)$, then $\mathcal{U}((V, A)) \leq \mathcal{U}((U, A))$, 
(SU3) For every $(U, A),(V, A) \in S(X \times X, A)$,

$$
\mathcal{U}((U, A) \odot(V, A)) \geq \mathcal{U}((U, A)) \odot \mathcal{U}((V, A))
$$

(SU4) If $\mathcal{U}((U, A)) \neq 0$, then $\left(1_{\triangle}, A\right) \leq(U, A)$, where

$$
1_{\Delta}(x, y)= \begin{cases}1 & \text { if } x=y \\ 0 & \text { if } x \neq y\end{cases}
$$

A soft $L$-fuzzy pre-uniformity $\mathcal{U}$ is called a soft $L$-fuzzy quasi-uniformity if

(Q) $\mathcal{U} \leq \mathcal{U} \circ \mathcal{U}$, where $\mathcal{U} \circ \mathcal{U}((U, A))=\bigvee\{\mathcal{U}((V, A)) \odot \mathcal{U}((W, A)) \mid(V, A) \circ$ $(W, A) \leq(U, A)\}$, for all $x, y \in X, a \in A$,

$$
\begin{aligned}
& (V(a) \circ W(a))(x, y) \\
& =\bigvee_{z \in X}(V(a)(z, x) \odot W(a)(x, y)) .
\end{aligned}
$$

The triple $(X, A, \mathcal{U})$ is called a soft $L$-fuzzy pre-(resp. quasi-) uniform space.

Remark 10. Let $(X, \mathcal{U})$ be a soft $L$-fuzzy quasi-uniform space, then by (SU1) and $(\mathrm{SU} 2)$, we have $\mathcal{U}\left(1_{X \times X}\right)=1$ because $(U, A) \leq\left(1_{X \times X}, A\right)$ for all $(U, A) \in S(X \times X, A)$.

\section{Soft $L$-Fuzzy Pre-Uniform Spaces Induced by Soft $L$-Fuzzy Topogenous Orders}

Lemma 11. For every $(F, A),(G, A) \in S(X, A)$, we define $\left(U_{F, G}, A\right)$, $\left(U_{F, G}^{-1}, A\right) \in S(X \times X, A)$ by

$$
\begin{gathered}
U_{F, G}(a)(x, y)=F(a)(x) \rightarrow G(a)(y) \\
U_{F, G}^{-1}(a)(x, y)=U_{F, G}(a)(y, x) .
\end{gathered}
$$

Then we have the following statements:

(1) $\left(1_{X \times X}, A\right)=\left(U_{0_{X}, 0_{X}}, A\right)=\left(U_{1_{X}, 1_{X}}, A\right)$,

(2) If $\left(F_{1}, A\right) \leq\left(F_{2}, A\right)$ and $\left(G_{1}, A\right) \leq\left(G_{2}, A\right)$, then $\left(U_{F_{2}, G_{1}}, A\right) \leq\left(U_{F_{1}, G_{2}}, A\right)$,

(3) If $(F, A) \leq(G, A)$, then $\left(1_{\triangle}, A\right) \leq\left(U_{F, G}, A\right)$,

(4) For every $\left(U_{F, G}, A\right) \in S(X \times X, A)$ and $(H, A) \in S(X, A)$, we have $\left(U_{H, G}, A\right) \circ\left(U_{F, H}, A\right) \leq\left(U_{F, G}, A\right)$. 
(5) $\left(U_{F_{1}, G_{1}}, A\right) \odot\left(U_{F_{2}, G_{2}}, A\right) \leq\left(U_{F_{1} \odot F_{2}, G_{1} \odot G_{2}}, A\right)$

(6) $\left(U_{F_{1}, G_{1}}, A\right) \odot\left(U_{F_{2}, G_{2}}, A\right) \leq\left(U_{F_{1} \oplus F_{2}, G_{1} \oplus G_{2}}, A\right)$

(7) $\left(U_{F, G}^{-1}, A\right)=\left(U_{G^{*}, F^{*}}, A\right)$

(8) $\left(U_{F_{1}, \odot F_{2}, G_{1} \odot G_{2}}^{-1}, A\right)=\left(U_{G_{1}^{*} \oplus G_{2}^{*}, F_{1}^{*} \oplus F_{2}^{*}}, A\right)$

(9) $\left(U_{F_{1} \oplus F_{2}, G_{1} \oplus G_{2}}^{-1}, A\right)=\left(U_{G_{1}^{*} \odot G_{2}^{*}, F_{1}^{*} \odot F_{2}^{*}}, A\right)$

Proof. (1)

$$
\begin{aligned}
& 1_{X \times X}(a)(x, y)=1=U_{0_{X}, 0_{X}}(a)(x, y)=0_{X}(a)(x) \rightarrow 0_{X}(a)(y) \\
& =1_{X}(a)(x) \rightarrow 1_{X}(a)(y)=U_{1_{X}, 1_{X}}(a)(x, y) .
\end{aligned}
$$

(2) Let $\left(F_{1}, A\right) \leq\left(F_{2}, A\right)$ and $\left(G_{1}, A\right) \leq\left(G_{2}, A\right)$.

$$
\begin{aligned}
& U_{F_{2}, G_{1}}(a)(x, y)=F_{2}(a)(x) \rightarrow G_{1}(a)(y) \\
& \leq F_{1}(a)(x) \rightarrow G_{2}(a)(y)=U_{F_{1}, G_{2}}(a)(x, y) .
\end{aligned}
$$

(3) Let $(F, A) \leq(G, A)$. We have $\left(1_{\triangle}, A\right) \leq\left(U_{F, G}, A\right)$ from

$$
U_{F, G}(a)(x, x)=F(a)(x) \rightarrow G(a)(x)=1 .
$$

(4)

$$
\begin{aligned}
& U_{H, G}(a)(x, z) \circ U_{F, H}(a)(x, z) \\
& =\bigvee_{y \in X}((H(a)(y) \rightarrow G(a)(z)) \odot(F(a)(x) \rightarrow H(a)(y))) \\
& \leq F(a)(x) \rightarrow G(a)(z)=U_{F, G}(a)(x, z) .
\end{aligned}
$$

(5) By Lemma 2(13), we have

$$
\begin{aligned}
& U_{F_{1}, G_{1}}(a)(x, y) \odot U_{F_{2}, G_{2}}(a)(x, y) \\
& =\left(F_{1}(a)(x) \rightarrow G_{1}(a)(y)\right) \odot\left(F_{2}(a)(x) \rightarrow G_{2}(a)(y)\right) \\
& \leq\left(F_{1}(a)(x) \odot F_{2}(a)(x) \rightarrow G_{1}(a)(y) \odot G_{2}(a)(y)\right. \\
& =U_{F_{1} \odot F_{2}, G_{1} \odot G_{2}}(a)(x, y) .
\end{aligned}
$$

(6) By Lemma 2(14), we have

$$
\begin{aligned}
& U_{F_{1}, G_{1}}(a)(x, y) \odot U_{F_{2}, G_{2}}(a)(x, y) \\
& =\left(F_{1}(a)(x) \rightarrow G_{1}(a)(y)\right) \odot\left(F_{2}(a)(x) \rightarrow G_{2}(a)(y)\right) \\
& \leq\left(F_{1}(a)(x) \oplus F_{2}(a)(x) \rightarrow G_{1}(a)(y) \oplus G_{2}(a)(y)\right. \\
& =U_{F_{1} \oplus F_{2}, G_{1} \oplus G_{2}(a)(x, y) .}
\end{aligned}
$$


(7) By Lemma 2(17), we have

$$
\begin{aligned}
& U_{F, G}^{-1}(a)(x, y)=U_{F, G}(a)(y, x)=F(a)(y) \rightarrow G(a)(x) \\
& =G(a)^{*}(x) \rightarrow F(a)^{*}(y)=U_{G^{*}, F^{*}}(a)(x, y) .
\end{aligned}
$$

(8)

$$
U_{F_{1} \odot F_{2}, G_{1} \odot G_{2}}^{-1}=U_{\left(G_{1} \odot G_{2}\right)^{*},\left(F_{1} \odot F_{2}\right)^{*}}=U_{G_{1}^{*} \oplus G_{2}^{*}, F_{1}^{*} \oplus F_{2}^{*}} .
$$

(9)

$$
U_{F_{1} \oplus F_{2}, G_{1} \oplus G_{2}}^{-1}=U_{\left(G_{1} \oplus G_{2}\right)^{*},\left(F_{1} \oplus F_{2}\right)^{*}}=U_{G_{1}^{*} \odot G_{2}^{*}, F_{1}^{*} \odot F_{2}^{*}} .
$$

Lemma 12. Let $(X, A, \mathcal{U})$ be a soft $L$-fuzzy quasi uniform space. For each $(U, A) \in S(X \times X, A)$ and $(F, A) \in S(X, A)$, the images $(U, A)[(F, A)],(U, A)[[(F$, $A)]]$ of $(F, A)$ with respect to $(U, A)$ are defined by, for all $x \in X, a \in A$,

$$
\begin{aligned}
(U, A)[(F, A)](a)(x) & =\bigvee_{y \in X}(F(a)(y) \odot U(a)(y, x)), \\
(U, A)[[(F, A)]](a)(x) & =\bigvee_{y \in X}(F(a)(y) \odot U(a)(x, y)) .
\end{aligned}
$$

For each $(U, A),(V, A),\left(U_{1}, A\right),\left(U_{2}, A\right) \in S(X \times X, A)$ and $(F, A),(G, A)$, $\left(F_{1}, A\right),\left(F_{2}, A\right),\left(F_{i}, A\right) \in S(X, A)$, we have 0

(1) $(F, A) \leq(U, A)[(F, A)]$ and $(F, A) \leq(U, A)[[(F, A)]]$ for each $\mathcal{U}((U, A))>$

(2) $(U, A) \leq(U, A) \circ(U, A)$, for each $\mathcal{U}((U, A))>0$,

(3) $((V, A) \circ(U, A))[(F, A)]=(V, A)[(U, A)[(F, A)]]((V, A) \circ(U, A))[[(F, A)]]$ $=(V, A)[[(U, A)[[(F, A)]]]]$,

(4) $(U, A)\left[\bigvee_{i}\left(F_{i}, A\right)\right]=\bigvee_{i}(U, A)\left[\left(F_{i}, A\right)\right]$ and $(U, A)\left[\left[\bigvee_{i}\left(F_{i}, A\right)\right]\right]$ $=\bigvee_{i}(U, A)\left[\left[\left(F_{i}, A\right)\right]\right]$

(5) $\left(\left(U_{1}, A\right) \odot\left(U_{2}, A\right)\right)\left[\left(F_{1}, A\right) \odot\left(F_{2}, A\right)\right] \leq\left(U_{1}, A\right)\left[\left(F_{1}, A\right)\right] \odot\left(U_{2}, A\right)\left[\left(F_{2}, A\right)\right]$,

(6) $\left(\left(U_{1}, A\right) \odot\left(U_{2}, A\right)\right)\left[\left[\left(F_{1}, A\right) \odot\left(F_{2}, A\right)\right]\right]$

$$
\leq\left(U_{1}, A\right)\left[\left[\left(F_{1}, A\right)\right]\right] \odot\left(U_{2}, A\right)\left[\left[\left(F_{2}, A\right)\right]\right],
$$

(7) $\left(\left(U_{1}, A\right) \odot\left(\left(U_{2}, A\right), A\right)\right)\left[\left(F_{1}, A\right) \oplus\left(F_{2}, A\right)\right]$

$$
\leq\left(U_{1}, A\right)\left[\left(F_{1}, A\right)\right] \oplus\left(\left(U_{2}, A\right), A\right)\left[\left(F_{2}, A\right)\right],
$$


(8) $\left(\left(U_{1}, A\right) \odot\left(\left(U_{2}, A\right), A\right)\right)\left[\left[\left(F_{1}, A\right) \oplus\left(F_{2}, A\right)\right]\right]$

$$
\leq\left(U_{1}, A\right)\left[\left[\left(F_{1}, A\right)\right]\right] \oplus\left(\left(U_{2}, A\right), A\right)\left[\left[\left(F_{2}, A\right)\right]\right] .
$$

(9) $\left(U_{F, G}, A\right)=\bigvee\{(W, A) \in S(X \times X, A) \mid(W, A)[(F, A)] \leq(G, A)\}$.

(10) $\left(U_{F, G}^{-1}, A\right)=\bigvee\{(W, A) \in S(X \times X, A) \mid(W, A)[[(F, A)]] \leq(G, A)\}$.

(11) $\left(U_{F, G}, A\right)[(F, A)] \leq(G, A)$ and $\left(U_{F, G}, A\right)\left[\left[(G, A)^{*}\right]\right] \leq(F, A)^{*}$. Moreover, $\left(U_{F, F}, A\right)[(F, A)]=(F, A)$ and $\left(U_{F, F}, A\right)\left[(F, A)^{*}\right]=(F, A)^{*}$.

Proof. (1)-(8) follows from [9].

(9) Since

$$
(W, A)[(F, A)](a)(y)=\bigvee_{x \in X}((W, A)(a)(x, y) \odot(F, A)(a)(x)) \leq(G, A)(y),
$$

then $(W, A)(a)(x, y) \leq F(a)(x) \rightarrow G(a)(y)=U_{F, G}(a)(x, y)$. So, $U_{F, G}(a)(x, y) \odot$ $(F, A)(a)(x)=(F(a)(x) \rightarrow G(a)(y)) \odot(F, A)(a)(x) \leq G(a)(y)$. Hence

$$
\left(U_{F, G}, A\right)=\bigvee\{(W, A) \in S(X \times X, A) \mid(W, A)[(F, A)] \leq(G, A)\} .
$$

(10) Since $(W, A)[[(F, A)]](a)(y)=\bigvee_{x \in X}((W, A)(a)(y, x) \odot(F, A)(a)(x))$ $\leq(G, A)(y)$, then $(W, A)(a)(y, x) \leq F(a)(x) \rightarrow G(a)(y)=U_{F, G}(a)(x, y)=$ $U_{F, G}^{-1}(a)(y, x)$. So, $U_{F, G}^{-1}(a)(y, x) \odot(F, A)(a)(x)=(F(a)(x) \rightarrow G(a)(y)) \odot$ $(F, A)(a)(x) \leq G(a)(y)$. Hence

$$
\left(U_{F, G}^{-1}, A\right)=\bigvee\{(W, A) \in S(X \times X, A) \mid(W, A)[[(F, A)]] \leq(G, A)\} .
$$

(11) Since

$$
\left(U_{F, G}, A\right)[(F, A)](a)(y)=\bigvee_{x \in X}((F(a)(x) \rightarrow G(a)(y)) \odot F(a)(x)) \leq G(a)(y),
$$

then $\left(U_{F, G}, A\right)[(F, A)] \leq G$. Since

$$
\begin{aligned}
& \left(U_{F, G}, A\right)\left[\left[\left(G^{*}, A\right)\right]\right](a)(x)=\bigvee_{y \in X}\left((F(a)(x) \rightarrow G(a)(y)) \odot G^{*}(a)(y)\right) \\
& =\bigvee_{y \in X}\left(\left(G^{*}(a)(y) \rightarrow F^{*}(a)(x)\right) \odot G^{*}(a)(y)\right) \leq F^{*}(a)(x),
\end{aligned}
$$

then $\left(U_{F, G}, A\right)\left[\left[(G, A)^{*}\right]\right] \leq(F, A)^{*}$. Moreover,

$$
\begin{aligned}
& \left(U_{F, F}, A\right)[(F, A)](a)(y) \geq U_{F, F}(y, y) \odot F(a)(y) \\
& (F(a)(y) \rightarrow F(a)(y)) \odot F(a)(y)=F(a)(y) .
\end{aligned}
$$




$$
\begin{aligned}
& \left(U_{F, F}, A\right)\left[\left[(F, A)^{*}\right]\right](a)(y) \geq U_{F, F}(y, y) \odot F^{*}(a)(y) \\
& \left(F^{*}(a)(y) \rightarrow F^{*}(a)(y)\right) \odot F^{*}(a)(y)=F^{*}(a)(y) .
\end{aligned}
$$

Theorem 13. [9] Let $(X, A, \mathcal{U})$ be a soft $L$-fuzzy quasi-uniform space. Define mappings $\xi_{\mathcal{U}}^{r}, \xi_{\mathcal{U}}^{l}: S(X, A) \times S(X, A) \rightarrow L$ by

$$
\begin{aligned}
& \xi_{\mathcal{U}}^{r}((F, A),(G, A))=\bigvee\{\mathcal{U}((U, A)) \mid(U, A)[(F, A)] \leq(G, A)\}, \\
& \xi_{\mathcal{U}}^{l}((F, A),(G, A))=\bigvee\{\mathcal{U}((U, A)) \mid(U, A)[[(F, A)]] \leq(G, A)\}
\end{aligned}
$$

Then $\xi_{\mathcal{U}}^{r}$ and $\xi_{\mathcal{U}}^{l}$ are soft L-fuzzy bitopogenous stuctures.

In the following theorem, we obtain a soft $L$-fuzzy pre-uniform structure from a soft $L$-fuzzy topogenous order.

Theorem 14. Let $\xi$ be a soft $L$-fuzzy topogenous order on $(X, A)$. Define $\mathcal{U}_{\xi}: S(X \times X, A) \rightarrow L$ by

$$
\mathcal{U}_{\xi}((U, A))=\bigvee\left\{\odot_{i=1}^{n} \xi\left(\left(F_{i}, A\right),\left(G_{i}, A\right)\right) \mid \odot_{i=1}^{n}\left(U_{F_{i}, G_{i}}, A\right) \leq(U, A)\right\},
$$

where $\bigvee$ is taken over every finite family $\left\{\left(U_{F_{i}, G_{i}}, A\right) \mid i=1,2,3, \ldots ., n\right\}$. Then

(1) $\mathcal{U}_{\xi}\left(\left(U_{\odot_{i=1}^{n} F_{i}, \odot_{i=1}^{n} G_{i}}, A\right)\right)=\xi\left(\odot_{i=1}^{n}\left(F_{i}, A\right), \odot_{i=1}^{n}\left(G_{i}, A\right)\right)$.

(2) $\mathcal{U}_{\xi}$ is a soft $L$-fuzzy pre-uniformity on $X$.

(3) If $\xi$ is a soft $L$-fuzzy topogenous structure on $(X, A)$, then $\mathcal{U}_{\xi}$ is a soft $L$-fuzzy quasi-uniformity on $X$.

(4) $\xi_{\mathcal{U}_{\xi}}^{r}=\xi$ and $\xi_{\mathcal{U}_{\xi}}^{l}=\xi^{s}$.

Proof. (1) Since $\odot_{i=1}^{n}\left(U_{F_{i}, G_{i}}, A\right) \leq\left(U_{\odot_{i=1}^{n}} F_{i}, \odot_{i=1}^{n} G_{i}, A\right)$ from Lemma 11 (5) and, by $(\mathrm{T})$,

$$
\begin{gathered}
\odot_{i=1}^{n} \xi\left(\left(F_{i}, A\right),\left(G_{i}, A\right)\right) \leq \xi\left(\odot_{i=1}^{n}\left(F_{i}, A\right), \odot_{i=1}^{n}\left(G_{i}, A\right)\right), \\
\mathcal{U}_{\xi}\left(\left(U_{\left.\left.\odot_{i=1}^{n} F_{i}, \odot_{i=1}^{n} G_{i}, A\right)\right)}=\xi\left(\odot_{i=1}^{n}\left(F_{i}, A\right), \odot_{i=1}^{n}\left(G_{i}, A\right)\right) .\right.\right.
\end{gathered}
$$

(2) (SU1) Since $\xi\left(\left(0_{X}, A\right),\left(0_{X}, A\right)\right)=\xi\left(\left(1_{X}, A\right),\left(1_{X}, A\right)\right)=1$, there exists $\left(1_{X \times X}, A\right)=\left(U_{0_{X}, 0_{X}}, A\right)=\left(U_{1_{X}, 1_{X}}, A\right) \in S(X \times X, A)$. It follows

$$
\mathcal{U}_{\xi}\left(\left(1_{X \times X}, A\right)\right)=1
$$

(SU2) It is trivial from the definition of $\mathcal{U}_{\xi}$. 
(SU3) For every $(U, A),(V, A) \in S(X \times X, A)$, each two families $\left\{U_{F_{i}, G_{i}} \mid \odot_{i=1}^{n}\right.$ $\left.U_{F_{i}, G_{i}} \leq U\right\}$ and $\left\{U_{H_{j}, K_{j}} \mid \odot_{j=1}^{k} U_{H_{j}, K_{j}} \leq V\right\}$, we have

$$
\begin{aligned}
& \mathcal{U}_{\xi}((U, A)) \odot \mathcal{U}_{\xi}((V, A)) \\
& =\left(\bigvee\left\{\odot_{i=1}^{n} \xi\left(\left(F_{i}, A\right),\left(G_{i}, A\right)\right) \mid \odot_{i=1}^{n}\left(U_{F_{i}, G_{i}}, A\right) \leq(U, A)\right\}\right) \\
& \odot\left(\bigvee\left\{\odot_{j=1}^{k} \xi\left(\left(H_{i}, A\right),\left(K_{i}, A\right)\right) \mid \odot_{j=1}^{k}\left(U_{H_{i}, K_{i}}, A\right) \leq(V, A)\right\}\right) \\
& \leq \bigvee\left\{\odot _ { i = 1 } ^ { n } \xi ( ( F _ { i } , A ) , ( G _ { i } , A ) ) \odot \left(\odot_{j=1}^{k} \xi\left(\left(H_{i}, A\right),\left(K_{i}, A\right)\right) \mid\right.\right. \\
& \left.\odot_{i=1}^{n}\left(U_{F_{i}, G_{i}}, A\right) \odot \odot_{j=1}^{k}\left(U_{H_{i}, K_{i}}, A\right) \leq(U, A) \odot(V, A)\right\} \\
& \leq \mathcal{U}_{\xi}((U, A) \odot(V, A)) .
\end{aligned}
$$

$(\mathrm{SU} 4)$ If $\mathcal{U}((U, A)) \neq 0$, there exists a family $\left\{\left(U_{F_{i}, G_{i}}, A\right) \mid \odot_{i=1}^{m}\left(U_{F_{i}, G_{i}}, A\right) \leq\right.$ $(U, A)\}$ such that

$$
\odot_{i=1}^{m} \xi\left(\left(F_{i}, A\right),\left(G_{i}, A\right)\right) \neq 0 .
$$

Since $\xi\left(\left(F_{i}, A\right),\left(G_{i}, A\right)\right) \neq 0$ for $i=1, \ldots m$, then $\left(F_{i}, A\right) \leq\left(G_{i}, A\right)$ for $i=1, \ldots m$, i.e. $\left(1_{\triangle}, A\right) \leq\left(U_{F_{i}, G_{i}}, A\right)$. Thus on $X$.

$\left(1_{\triangle}, A\right) \leq \odot_{i=1}^{m}\left(U_{F_{i}, G_{i}}, A\right) \leq(U, A)$. Then $\mathcal{U}_{\xi}$ is a soft $L$-fuzzy pre-uniformity

(3) Let $\xi$ be a soft $L$-fuzzy topogenous structure on $(X, A)$.

(Q) Suppose there exists $(U, A) \in S(X \times X, A)$ such that

$$
\begin{aligned}
& t=\bigvee\left\{\mathcal{U}_{\xi}((V, A)) \odot \mathcal{U}_{\xi}((W, A)) \mid(V, A) \circ(W, A) \leq(U, A)\right\} \\
& \nsupseteq \mathcal{U}_{\xi}((U, A)) .
\end{aligned}
$$

From the definition of $\mathcal{U}_{\xi}((U, A))$, there exists a family

$$
\left\{\left(U_{F_{i}, G_{i}}, A\right) \mid \odot_{i=1}^{m}\left(U_{F_{i}, G_{i}}, A\right) \leq(U, A)\right\}
$$

such that

$$
t \geq \odot_{i=1}^{m} \xi\left(\left(F_{i}, A\right),\left(G_{i}, A\right)\right) .
$$

Since $\xi \circ \xi \geq \xi$

$$
\begin{aligned}
t & \nsupseteq \odot_{i=1}^{m}(\xi \circ \xi)\left(\left(F_{i}, A\right),\left(G_{i}, A\right)\right) \\
& =\odot_{i=1}^{m}\{\bigvee(H, A) \in S(X, A) \\
& \left(\xi\left((H, A),\left(G_{i}, A\right)\right) \odot \xi\left(\left(F_{i}, A\right),(H, A)\right)\right\} .
\end{aligned}
$$

Since $L$ is a complete residuated lattice, there exists $\left(H_{i}, A\right) \in S(X, A)$ such that

$$
t \nsupseteq \odot_{i=1}^{m}\left(\xi\left(\left(H_{i}, A\right),\left(G_{i}, A\right)\right) \odot \xi\left(\left(F_{i}, A\right),\left(H_{i}, A\right)\right)\right) .
$$

On the other hand, put $\left(V_{i}, A\right)=\left(U_{H_{i}, G_{i}}, A\right),\left(W_{i}, A\right)=\left(U_{F_{i}, H_{i}}, A\right)$. From Lemma 11(4), it satisfies

$$
\left(V_{i}, A\right) \circ\left(W_{i}, A\right)=\left(U_{H_{i}, G_{i}}, A\right) \circ\left(U_{F_{i}, H_{i}}, A\right) \leq\left(U_{F_{i}, G_{i}}, A\right),
$$




$$
\begin{aligned}
& \mathcal{U}_{\xi}\left(\left(V_{i}, A\right)\right) \geq \xi\left(\left(H_{i}, A\right),\left(G_{i}, A\right)\right), \\
& \mathcal{U}_{\xi}\left(\left(W_{i}, A\right)\right) \geq \xi\left(\left(F_{i}, A\right),\left(H_{i}, A\right)\right) .
\end{aligned}
$$

Let $(V, A)=\odot_{i=1}^{m}\left(V_{i}, A\right)$ and $(W, A)=\odot_{i=1}^{m}\left(W_{i}, A\right)$ be given. Since $\left(V_{i}, A\right) \circ$ $\left(W_{i}, A\right) \leq\left(U_{F_{i}, G_{i}}, A\right)$ for each $i=1, \ldots, m$, we have

$$
\begin{aligned}
& \left(\odot_{i=1}^{m}\left(V_{i}, A\right)\right) \circ\left(\odot_{i=1}^{m}\left(W_{i}, A\right)\right)=\odot_{i=1}^{m}\left(\left(V_{i}, A\right) \circ\left(W_{i}, A\right)\right) \\
& \leq \odot_{i=1}^{m}\left(U_{F_{i}, G_{i}}, A\right) \leq(U, A) .
\end{aligned}
$$

Then we have $(V, A) \circ(W, A) \leq(U, A)$ and $\mathcal{U}_{\xi}((V, A)) \geq \odot_{i=1}^{m} \mathcal{U}_{\xi}\left(\left(V_{i}, A\right)\right)$ and $\mathcal{U}_{\xi}((W, A)) \geq \odot_{i=1}^{m} \mathcal{U}_{\xi}\left(\left(W_{i}, A\right)\right)$. Thus,

$$
\begin{aligned}
& t=\bigvee\left\{\mathcal{U}_{\xi}((V, A)) \odot \mathcal{U}_{\xi}((W, A)) \mid(V, A) \circ(W, A) \leq(U, A)\right\} \\
& \geq \mathcal{U}_{\xi}((V, A)) \odot \mathcal{U}_{\xi}((W, A)) \\
& \geq \odot_{i=1}^{m} \mathcal{U}_{\xi}\left(\left(V_{i}, A\right)\right) \odot \odot_{i=1}^{m} \mathcal{U}_{\xi}\left(\left(W_{i}, A\right)\right) \\
& \geq \odot_{i=1}^{m}\left(\xi\left(\left(H_{i}, A\right),\left(G_{i}, A\right)\right) \odot \xi\left(\left(F_{i}, A\right),\left(H_{i}, A\right)\right) .\right.
\end{aligned}
$$

It is a contradiction for the equation (I).

Then $\mathcal{U}_{\xi}$ is a soft $L$-fuzzy quasi uniformity on $X$.

(4)

$$
\begin{aligned}
& \xi_{\mathcal{U}_{\xi}}^{r}((F, A),(G, A))=\bigvee\left\{\mathcal{U}_{\xi}((U, A)) \mid(U, A)[(F, A)] \leq(G, A)\right\} \\
& =\mathcal{U}_{\xi}\left(U_{F, G}\right)=\xi((F, A),(G, A)) . \\
& \xi_{\mathcal{U}_{\xi}}^{l}((F, A),(G, A))=\bigvee\left\{\mathcal{U}_{\xi}((U, A)) \mid(U, A)[[(F, A)]] \leq(G, A)\right\} \\
& =\mathcal{U}_{\xi}\left(U_{F, G}^{-1}\right)=\xi^{s}((F, A),(G, A)) .
\end{aligned}
$$

Theorem 15. Let $\xi$ be a soft $L$-fuzzy cotopogenous order on $(X, A)$. Define $\mathcal{U}_{\xi}: S(X \times X, A) \rightarrow L$ by

$$
\begin{aligned}
\mathcal{U}_{\xi}((U, A)) & =\bigvee\left\{\odot_{i=1}^{n} \xi\left(\left(F_{i}, A\right),\left(G_{i}, A\right)\right) \mid\right. \\
& \left.\odot_{i=1}^{n}\left(U_{F_{i}, G_{i}}, A\right) \leq(U, A)\right\},
\end{aligned}
$$

where $\bigvee$ is taken over every finite family $\left\{\left(U_{F_{i}, G_{i}}, A\right) \mid i=1,2,3, \ldots ., n\right\}$. Then

(1) $\mathcal{U}_{\xi}\left(\left(U_{\oplus_{i=1}^{n} F_{i}, \oplus_{i=1}^{n} G_{i}}, A\right)\right)=\xi\left(\oplus_{i=1}^{n}\left(F_{i}, A\right), \oplus_{i=1}^{n}\left(G_{i}, A\right)\right)$.

(2) $\mathcal{U}_{\xi}$ is a soft $L$-fuzzy pre-uniformity on $X$.

(3) If $\xi$ is a soft $L$-fuzzy cotopogenous structure on $(X, A)$, then $\mathcal{U}_{\xi}$ is a soft $L$-fuzzy quasi-uniformity on $X$.

(4) $\mathcal{U}_{\xi^{s}}((U, A))=\mathcal{U}_{\xi}\left((U, A)^{-1}\right)$ for all $(U, A) \in S(X \times X, L)$.

(5) $\xi_{\mathcal{U}_{\xi}}^{r}=\xi$ and $\xi_{\mathcal{U}_{\xi}}^{l}=\xi^{s}$. 
Proof. (1) Since $\odot_{i=1}^{n}\left(U_{F_{i}, G_{i}}, A\right) \leq\left(U_{\oplus_{i=1}^{n}} F_{i}, \oplus_{i=1}^{n} G_{i}, A\right)$ from Lemma 11 (6) and, by (CT),

$$
\begin{aligned}
\odot_{i=1}^{n} \xi( & \left.\left(F_{i}, A\right),\left(G_{i}, A\right)\right) \leq \xi\left(\oplus_{i=1}^{n}\left(F_{i}, A\right), \oplus_{i=1}^{n}\left(G_{i}, A\right)\right), \text { then } \\
& \mathcal{U}_{\xi}\left(\left(U_{\oplus_{i=1}^{n}} F_{i}, \oplus_{i=1}^{n} G_{i}, A\right)\right)=\xi\left(\oplus_{i=1}^{n}\left(F_{i}, A\right), \oplus_{i=1}^{n}\left(G_{i}, A\right)\right) .
\end{aligned}
$$

$$
\begin{aligned}
& \mathcal{U}_{\xi^{s}}((U, A))=\bigvee\left\{\odot_{i=1}^{n} \xi^{s}\left(\left(F_{i}, A\right),\left(G_{i}, A\right)\right) \mid \odot_{i=1}^{n}\left(U_{F_{i}, G_{i}}, A\right) \leq(U, A)\right\} \\
& =\bigvee\left\{\odot_{i=1}^{n} \xi\left(\left(G_{i}^{*}, A\right),\left(F_{i}^{*}, A\right)\right) \mid \odot_{i=1}^{n}\left(U_{F_{i}, G_{i}}^{-1}, A\right) \leq(U, A)^{-1}\right\} \\
& =\bigvee\left\{\odot_{i=1}^{n} \xi\left(\left(G_{i}^{*}, A\right),\left(F_{i}^{*}, A\right)\right) \mid \odot_{i=1}^{n}\left(U_{G_{i}^{*}, F_{i}^{*}}, A\right) \leq(U, A)^{-1}\right\} \\
& =\mathcal{U}_{\xi}\left((U, A)^{-1}\right) .
\end{aligned}
$$

(2), (3) and (5) are similarly proved as Theorem 14.

Example 16. Let $U=\left\{h_{i} \mid i=\{1, \ldots, 6\}\right\}$ with $h_{i}=$ house and $E=$ $\{e, b, w, c, i\}$ with $e=$ expensive, $b=$ beautiful, $w=$ wooden, $c=$ creative, $i=$ in the green surroundings.

Define a binary operation $\odot$ on $[0,1]$ by

$$
\begin{gathered}
x \odot y=\max \{0, x+y-1\}, \quad x \rightarrow y=\min \{1-x+y, 1\}, \\
x \oplus y=\min \{1, x+y\}, \quad x^{*}=1-x .
\end{gathered}
$$

Then $([0,1], \wedge, \rightarrow, 0,1)$ is a complete residuated lattice (ref.[4.5]). Let $A=$ $\{b, c, i\} \subset E$ and $X=\left\{h^{1}, h^{4}, h^{5}, h^{6}\right\}$. Put $(H, A)$ be a fuzzy soft set as follow:

$$
\begin{array}{cccccc}
(H, A) & h^{1} & h^{4} & h^{5} & h^{6} & \\
b & 0.5 & 0.6 & 0.2 & 0.6 & \\
c & 0.1 & 0.5 & 0.5 & 0.6 & \\
i & 0.4 & 0.6 & 0.6 & 0.5 & \\
(H, A) \odot(H, A) & h^{1} & h^{4} & h^{5} & h^{6} \\
b & & 0.0 & 0.2 & 0.0 & 0.2 \\
c & & 0.0 & 0.0 & 0.0 & 0.2 \\
i & & 0.0 & 0.2 & 0.2 & 0.0 \\
\left(H^{*}, A\right) & h^{1} & h^{4} & h^{5} & h^{6} & \\
b & 0.5 & 0.4 & 0.8 & 0.4 & \\
c & 0.9 & 0.5 & 0.5 & 0.4 & \\
i & 0.6 & 0.4 & 0.4 & 0.5 &
\end{array}
$$




$$
\begin{array}{ccccc}
\left(H^{*}, A\right) \oplus\left(H^{*}, A\right) & h^{1} & h^{4} & h^{5} & h^{6} \\
b & 1.0 & 0.8 & 1.0 & 0.8 \\
c & 1.0 & 1.0 & 1.0 & 0.8 \\
i & 1.0 & 0.8 & 0.8 & 1.0
\end{array}
$$

(1) Define a soft $L$-fuzzy topogenous order $\xi: S(X, A) \times S(X, A) \rightarrow L$ as follows

$$
\xi((F, A),(G, A))= \begin{cases}1, & \text { if }(F, A)=(\overline{0}, A) \text { or }(G, A)=(\overline{1}, A) \\ 0.6, \quad \text { if }(F, A) \leq(H, A) \leq(G, A), & (F, A) \not \leq(H, A) \odot(H, A) \\ 0.3, \quad \text { if }(\overline{0}, A) \neq(F, A) \leq(H, A) \odot(H, A) & \leq(G, A),(H, A) \not \leq(G, A), \\ 0, & \text { otherwise, }\end{cases}
$$

But it is not a soft $L$-fuzzy topogenous structure because

$$
\begin{aligned}
& \bigvee_{(F, A) \in S(X, A)}(\xi((H, A) \odot(H, A),(F, A)) \\
& \odot \xi((F, A),(H, A) \odot(H, A)))=0 \\
& \geq \xi((H, A) \odot(H, A),(H, A) \odot(H, A))=0.3 .
\end{aligned}
$$

We obtain $\left(U_{H, H}, A\right),\left(U_{H \odot H, H \odot H}, A\right) \in S(X \times X, A)$ such that, for $a \in A$, $U_{H, H}(a) \in L^{X \times X}$ with $U_{H, H}(a)(x, y)=H(a)(x) \rightarrow H(a)(y)$,

$$
\begin{aligned}
U_{H, H}(b) & =\left(\begin{array}{cccc}
1 & 1 & 0.7 & 1 \\
0.9 & 1 & 0.6 & 1 \\
1 & 1 & 1 & 1 \\
0.9 & 1 & 0.6 & 1
\end{array}\right), U_{H, H}(c)=\left(\begin{array}{ccccc}
1 & 1 & 1 & 1 \\
0.6 & 1 & 1 & 1 \\
0.6 & 1 & 1 & 1 \\
0.5 & 0.9 & 0.9 & 1
\end{array}\right) \\
U_{H, H}(i) & =\left(\begin{array}{cccc}
1 & 1 & 1 & 1 \\
0.8 & 1 & 1 & 0.9 \\
0.8 & 1 & 1 & 0.9 \\
0.9 & 1 & 1 & 1
\end{array}\right), U_{H \odot H, H \odot H}(b)=\left(\begin{array}{ccccc}
1 & 1 & 1 & 1 \\
0.8 & 1 & 0.8 & 1 \\
1 & 1 & 1 & 1 \\
0.8 & 0.8 & 1
\end{array}\right) \\
U_{H \odot H, H \odot H}(c) & =\left(\begin{array}{cccc}
1 & 1 & 1 \\
1 & 1 & 1 & 1 \\
1 & 1 & 1 & 1 \\
0.8 & 0.8 & 0.8 & 1
\end{array}\right), U_{H \odot H, H \odot H}(i)=\left(\begin{array}{cccc}
1 & 1 & 1 \\
0.8 & 1 & 1 & 0.8 \\
0.8 & 1 & 1 & 0.8 \\
1 & 1 & 1 & 1
\end{array}\right)
\end{aligned}
$$

By Theorem 14, we obtain a soft $L$-fuzzy pre-uniformity $\mathcal{U}_{\xi}: S(X \times X, A) \rightarrow$ 
$L$ as follows

$$
\mathcal{U}_{\xi}((U, A))= \begin{cases}1, & \text { if }(U, A)=\left(1_{X \times X}, A\right), \\ 0.6, & \text { if }\left(U_{H, H}, A\right) \leq(U, A) \neq\left(1_{X \times X}, A\right), \\ 0.3, & \text { if }\left(U_{H \odot H, H \odot H}, A\right) \leq(U, A) \\ & (U, A) \geq\left(U_{H, H}, A\right), \\ 0.2, & \text { if }\left(U_{H, H}, A\right) \odot\left(U_{H, H}, A\right) \leq(U, A), \\ & (U, A) \geq\left(U_{H \odot H, H \odot H}, A\right), \\ 0, & \text { otherwise. }\end{cases}
$$

Since $H(b) \circ H(b)=H(b)$, we have

$$
\begin{aligned}
& \mathcal{U}_{\xi} \circ \mathcal{U}_{\xi}\left(\left(U_{H, H}, A\right) \odot\left(U_{H, H}, A\right)\right)=0 \\
& \neq 0.2=\mathcal{U}_{\xi}\left(\left(U_{H, H}, A\right) \odot\left(U_{H, H}, A\right)\right) .
\end{aligned}
$$

Hence $\mathcal{U}_{\xi}$ is not a soft $L$-fuzzy quasi-uniformity.

(2) By Remark 6 and (1), we obtain a soft $L$-fuzzy cotopogenous order $\xi^{s}: S(X, A) \times S(X, A) \rightarrow L$ as follows

$$
\xi^{s}((F, A),(G, A))= \begin{cases}1, & \text { if }(F, A)=(\overline{0}, A) \text { or }(G, A)=(\overline{1}, A) \\ 0.6, & \text { if }(F, A) \leq(H, A)^{*} \leq(G, A), \\ & (G, A) \geq(H, A)^{*} \oplus(H, A)^{*} \\ 0.3, & \text { if }(F, A) \leq(H, A)^{*} \oplus(H, A)^{*} \\ & \leq(G, A) \neq(\overline{1}, A),(F, A) \not \leq(H, A)^{*}, \\ 0, & \text { otherwise, }\end{cases}
$$

But it is not a soft $L$-fuzzy cotopogenous structure because

$$
\begin{aligned}
& \bigvee_{(F, A) \in S(X, A)}\left(\xi^{s}\left((H, A)^{*} \oplus(H, A)^{*},(F, A)\right)\right. \\
& \left.\odot \xi^{s}\left((F, A),(H, A)^{*} \oplus(H, A)^{*}\right)\right)=0 \\
& \geq \xi^{s}\left((H, A)^{*} \oplus(H, A)^{*},(H, A)^{*} \oplus(H, A)^{*}\right)
\end{aligned}
$$

We obtain $\left(U_{H, H}, A\right),\left(U_{H \odot H, H \odot H}, A\right) \in S(X \times X, A)$ such that, for $a \in A$, $U_{H, H}(a) \in L^{X \times X}$ with $U_{H, H}(a)(x, y)=H(a)(x) \rightarrow H(a)(y)$,

$$
\begin{aligned}
& U_{H^{*}, H^{*}}(b)=\left(\begin{array}{cccc}
1 & 0.9 & 1 & 0.9 \\
1 & 1 & 1 & 1 \\
0.7 & 0.6 & 1 & 0.6 \\
1 & 1 & 1 & 1
\end{array}\right), U_{H^{*}, H^{*}}(c)=\left(\begin{array}{cccc}
1 & 0.6 & 0.6 & 0.5 \\
1 & 1 & 1 & 0.9 \\
1 & 1 & 1 & 0.9 \\
1 & 1 & 1 & 1
\end{array}\right) \\
& U_{H^{*}, H^{*}}(i)=\left(\begin{array}{cccc}
1 & 0.8 & 0.8 & 0.9 \\
1 & 1 & 1 & 1 \\
1 & 1 & 1 & 1 \\
1 & 0.9 & 0.9 & 1
\end{array}\right), U_{H^{*} \oplus H^{*}, H^{*} \oplus H^{*}}(b)=\left(\begin{array}{cccc}
1 & 0.8 & 1 & 0.8 \\
1 & 1 & 1 & 1 \\
1 & 0.8 & 1 & 0.8 \\
1 & 1 & 1 & 1
\end{array}\right)
\end{aligned}
$$




$$
\begin{aligned}
U_{H^{*} \oplus H^{*}, H^{*} \oplus H^{*}}(c) & =\left(\begin{array}{cccc}
1 & 1 & 1 & 0.8 \\
1 & 1 & 1 & 0.8 \\
1 & 1 & 1 & 0.8 \\
1 & 1 & 1 & 1
\end{array}\right), \\
U_{H^{*} \oplus H^{*}, H^{*} \oplus H^{*}}(i) & =\left(\begin{array}{cccc}
1 & 0.8 & 0.8 & 1 \\
1 & 1 & 1 & 1 \\
1 & 1 & 1 & 1 \\
1 & 0.8 & 0.8 & 1
\end{array}\right)
\end{aligned}
$$

By Theorem 15, we obtain a soft $L$-fuzzy pre-uniformity $\mathcal{U}_{\xi^{s}}: S(X \times$ $X, A) \rightarrow L$ as follows

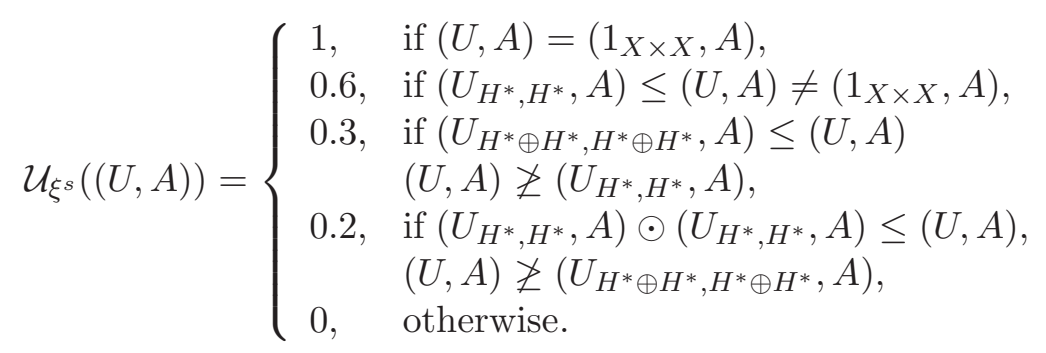

Since $H^{*}(b) \circ H^{*}(b)=H^{*}(b)$, we have

$$
\begin{aligned}
& \mathcal{U}_{\xi^{s}} \circ \mathcal{U}_{\xi^{s}}\left(\left(U_{H^{*}, H^{*}}, A\right) \odot\left(U_{H^{*}, H^{*}}, A\right)\right)=0 \\
& \neq 0.2=\mathcal{U}_{\xi^{s}}\left(\left(U_{H^{*}, H^{*}}, A\right) \odot\left(U_{H^{*}, H^{*}}, A\right)\right) .
\end{aligned}
$$

Hence $\mathcal{U}_{\xi^{s}}$ is not a soft $L$-fuzzy quasi-uniformity.

\section{References}

[1] K.V. Babitha, J.J. Sunil, Soft set relations and functions, Compu. Math. Appl., 60 (2010), 1840-1849, doi: 10.1016/j.camwa.2010.07.014.

[2] N. Cag̈man, S. Karatas and S. Enginoglu, Soft topology, Comput. Math. Appl., 62 (1) (2011), 351-358. doi: 10.1016/j.camwa.2011.05.016.

[3] F. Feng, X. Liu, V.L. Fotea, Y.B. Jun, Soft sets and soft rough sets, Information Sciences, 181 (2011), 1125-1137, doi: 10.1016/j.ins.2010.11.004.

[4] P. Hájek, Metamathematices of Fuzzy Logic, Kluwer Academic Publishers, Dordrecht (1998), doi: 10.1007/978-94-011-5300-3.

[5] U. Höhle, S.E. Rodabaugh, Mathematics of Fuzzy Sets: Logic, Topology, and Measure Theory, The Handbooks of Fuzzy Sets Series 3, Kluwer Academic Publishers, Boston, 1999, doi: 10.1007/978-1-4615-5079-2.

[6] A.K. Katsaras, On fuzzy uniform spaces, J. Math. Anal. Appl., 101, 1984, 97-113. doi: 10.1016/0022-247x(84)90060-x. 
[7] J.M. Ko, Y.C. Kim, Soft $L$-topologies and soft $L$-neighborhood systems, (accepted to) J. Math. Comput. Sci.

[8] J.M. Ko, Y.C. Kim, Soft $L$-uniformities and soft $L$-neighborhood systems, (accepted to) J. Math. Comput. Sci.

[9] J.M. Ko, Y.C. Kim, Soft $L$-fuzzy quasi-uniformities and soft $L$-fuzzy topogenous orders, (submit to) Int. J. of Pure and Applied Math.

[10] R. Lowen, Fuzzy uniform spaces, J. Math. Anal. Appl., 82 (1981), 370-385, doi: 10.1016/0022-247x(81)90202-x.

[11] D. Molodtsov, Soft set theory, Comput. Math. Appl., 37 (1999), 19-31.

[12] Z. Pawlak, Rough sets, Int. J. Comput. Inf. Sci., 11 (1982), 341-356.

[13] Z. Pawlak, Rough probability, Bull. Pol. Acad. Sci. Math., 32 (1984), 607-615.

[14] M. Shabir, M. Naz, On soft topological spaces, Comput. Math. Appl.,61 (2011), 17861799, doi: 10.1016/j.camwa.2011.02.006.

[15] B. Tanay, M. B. Kandemir, Topological structure of fuzzy soft sets, Comput. Math. Appl., 61 (10) (2011), 2952-2957, doi: 10.1016/j.camwa.2011.03.056.

[16] Hu Zhao and Sheng-Gang Li, L-fuzzifying soft topological spaces and L-fuzzifying soft interior operators, Ann. Fuzzy Math. Inform., 5 (3) (2013), 493-503.

[17] Í. Zorlutuna, M. Akdag, W. K. Min, S. Atmaca, Remarks on soft topological spaces, Ann. Fuzzy Math. Inform., 3 (2) (2012), 171-185. 